ARTICLE

\title{
Subtraction-free and bisulfite-free specific sequencing of 5-methylcytosine and its oxidized derivatives at base resolution
}

Yibin Liu 1,2,5,6, Zhiyuan Hu (10 3,4,6, Jingfei Cheng ${ }^{1,2}$, Paulina Siejka-Zielińska ${ }^{1,2}$, Jinfeng Chen ${ }^{1,2}$, Masato Inoue ${ }^{1,2}$,

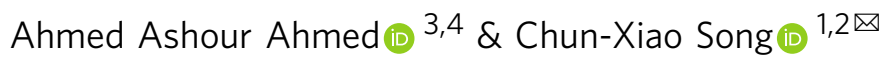

Although various methods have been developed for sequencing cytosine modifications, it is still challenging for specific and quantitative sequencing of individual modification at baseresolution. For example, to obtain both true 5-methylcytosine $(5 \mathrm{mC})$ and true 5hydroxymethylcytosine $(5 \mathrm{hmC})$ information, the two major epigenetic modifications, it usually requires subtraction of two methods, which increases noise and requires high sequencing depth. Recently, we developed TET-assisted pyridine borane sequencing (TAPS) for bisulfite-free direct sequencing of $5 \mathrm{mC}$ and $5 \mathrm{hmC}$. Here we demonstrate that two sister methods, TAPS $\beta$ and chemical-assisted pyridine borane sequencing (CAPS), can be effectively used for subtraction-free and specific whole-genome sequencing of $5 \mathrm{mC}$ and $5 \mathrm{hmC}$, respectively. We also demonstrate pyridine borane sequencing (PS) for whole-genome profiling of 5-formylcytosine and 5-carboxylcytosine, the further oxidized derivatives of $5 \mathrm{mC}$ and $5 \mathrm{hmC}$. This work completes the set of versatile borane reduction chemistry-based methods as a comprehensive toolkit for direct and quantitative sequencing of all four cytosine epigenetic modifications.

\footnotetext{
${ }^{1}$ Ludwig Institute for Cancer Research, Nuffield Department of Medicine, University of Oxford, Oxford OX3 7FZ, UK. ${ }^{2}$ Target Discovery Institute, Nuffield Department of Medicine, University of Oxford, Oxford OX3 7FZ, UK. ${ }^{3}$ Ovarian Cancer Cell Laboratory, MRC Weatherall Institute of Molecular Medicine, University of Oxford, Oxford OX3 9DS, UK. ${ }^{4}$ Nuffield Department of Women's \& Reproductive Health, University of Oxford, Oxford OX3 9DU, UK. ${ }^{5}$ Present address: Exact Sciences Innovation, Innovation Building, Oxford OX3 7FZ, UK. ${ }^{6}$ These authors contributed equally: Yibin Liu, Zhiyuan Hu. ${ }^{凶}$ email: chunxiao.song@ludwig.ox.ac.uk
} 
T he primary DNA sequence of the four-letter alphabets G, C, $\mathrm{A}$, and $\mathrm{T}$ forms the genetic information of life on earth. Chemical modifications of DNA bases do not change the underlying sequence, but instead carry an extra layer of information. The first discovered 5-methylcytosine $(5 \mathrm{mC})$ is the most studied modified base, and it plays crucial roles in a broad range of biological processes from gene regulation to normal development ${ }^{1}$ and is regarded as the fifth base. 5-Hydroxymethylcytosine $(5 \mathrm{hmC})$ is converted from $5 \mathrm{mC}$ by the ten-eleven translocation (TET) family of dioxygenases ${ }^{2}$; it is enriched in neuronal cells ${ }^{3}$ and regarded as the sixth base. Further successive TET oxidation results in 5-formylcytosine (5fC) and 5-carboxylcytosine $(5 \mathrm{caC})^{4,5}$, which exist at much lower abundances in the mammalian genome and are regarded as intermediates in the thymine DNA glycosylase (TDG)-base excision repair (BER) active demethylation pathway ${ }^{5}$. Emerging evidence indicates the stability of these DNA demethylation intermediates ${ }^{6}$ as well as potential functional roles ${ }^{7}$.

Detection and analysis of cytosine modifications has been an intriguing challenge for chemists as well as other scientists. Traditionally, bisulfite sequencing (BS) has been the gold standard for base-resolution and quantitative analysis of $5 \mathrm{mC}$ and $5 \mathrm{hmC}$. Modified BS has also been developed for specific sequencing of $5 \mathrm{mC}$ (oxidative bisulfite sequencing, oxBS-seq) $)^{9}$ or $5 \mathrm{hmC}$ (TETassisted bisulfite sequencing, TAB-seq) ${ }^{10}$. These methods, however, all involve harsh bisulfite treatment, which degrades up to $99 \%$ of the $\mathrm{DNA}^{11}$, and reduces sequence complexity by converting unmodified cytosine ( $\sim 95 \%$ of the total cytosine in the human genome) to thymine (T). Recently, bisulfite-free quantitative base-resolution methods have emerged and showed significant advantages over $\mathrm{BS}^{12}$. Among them, APOBEC-coupled epigenetic sequencing (ACE-seq, which detects $5 \mathrm{hmC})^{13}$ and Enzymatic Methyl-seq (EM-seq, which detects $5 \mathrm{mC}+5 \mathrm{hmC})^{14}$ use an enzymatic deamination step to replace the bisulfite deamination step. While these methods solve the DNA damage issue, they still suffer from the indirect detection issue of BS by converting unmodified cytosine to T. Recently, we developed TET-assisted pyridine borane sequencing (TAPS) based on a pyridine borane reductive decarboxylation and deamination chemistry ${ }^{15,16}$. In TAPS, $5 \mathrm{mC}$ and $5 \mathrm{hmC}$ are oxidized by TET proteins to $5 \mathrm{caC}$ and reduced to dihydrouracil (DHU) by pyridine borane. DHU is then amplified and sequenced as T during sequencing. TAPS is nondestructive and detects $5 \mathrm{mC}+5 \mathrm{hmC}$ directly, and it shows improved sequence quality, mapping rate, and coverage compared to $\mathrm{BS}^{15}$.

$5 \mathrm{mC}$ and $5 \mathrm{hmC}$ provide distinct and antagonistic epigenetic information: $5 \mathrm{mC}$ usually marks repressed genes and $5 \mathrm{hmC}$ generally marks expressed genes ${ }^{17}$. To elucidate the interplay between $5 \mathrm{mC}$ and $5 \mathrm{hmC}$ in various biological processes, it is necessary to distinguish the two modifications. To do that, two assays (e.g. BS and oxBS-seq, BS and TAB-seq, or EM-Seq and ACE-seq) need to be performed and a subtraction between the two assays is usually required to obtain both the true $5 \mathrm{mC}$ and true $5 \mathrm{hmC}$ information (e.g. BS minus oxBS-seq to get $5 \mathrm{hmC}$, BS minus TAB-seq to get $5 \mathrm{mC}$, or EM-Seq minus ACE-seq to get $5 \mathrm{mC})^{9,10,13}$. However, subtraction may introduce negative values because of random sampling or systematic error in each experiment and suffer from accumulation of noise from multiple assays, which increases the need for higher sequencing depth ${ }^{18}$ as well as more effort to perform read filtering and apply statistical tests ${ }^{19}$. A subtraction-free approach where two assays (e.g. oxBS-seq and TAB-seq) can read out the true $5 \mathrm{mC}$ and true $5 \mathrm{hmC}$ information directly is desirable. However, so far, no bisulfite-free methods have been shown to deliver that. Previously, we demonstrated the proof-of-principle that the versatility of the borane reduction chemistry for direct and quantitative sequencing of individual cytosine modification on model DNA with Sanger sequencing ${ }^{15}$.
In this study, we further optimize and demonstrate these methods for whole-genome applications, including TAPS with $\beta$ glucosyltransferase ( $\beta \mathrm{GT}$ ) blocking (TAPS $\beta$ ) and chemicalassisted pyridine borane sequencing (CAPS) for whole-genome subtraction-free $5 \mathrm{mC}$-specific and $5 \mathrm{hmC}$-specific sequencing, respectively; and pyridine borane sequencing (PS) for wholegenome sequencing of $5 \mathrm{fC}$ and $5 \mathrm{caC}$.

\section{Results}

TAPS $\beta$ for bisulfite-free $5 \mathrm{mC}$-specific sequencing. To realize $5 \mathrm{mC}$-specific sequencing, we used $\beta \mathrm{GT}$, which is widely used for selective labeling of $5 \mathrm{hmC}$ with glucose that enables $5 \mathrm{hmC}$ pulldown ${ }^{20}$ and protection from TET oxidation ${ }^{10}$ or APOBEC deamination ${ }^{13}$. We utilized this simple and robust reaction to block $5 \mathrm{hmC}$ and then performed TET oxidation and borane reduction on $5 \mathrm{mC}$ (Fig. 1a) ${ }^{15}$. We applied TAPS $\beta$ on mouse embryonic stem cells (mESCs) genomic DNA (gDNA) and validated with spike-in controls with known modifications by high-throughput sequencing. High $5 \mathrm{mC}$ conversion rate $(97.6 \%$ in $\mathrm{CpG}-$ methylated lambda DNA, Fig. 1b) and low false-positive rate $(0.24 \%$ conversion rate on unmodified C, Fig. 1c) were achieved in TAPS $\beta$, which are close to previous TAPS results $(96.5 \%$ and $0.23 \%$, respectively $)^{15} .5 \mathrm{hmC}$ showed only $1.9 \%$ conversion rate in TAPS $\beta$ (Fig. 1b) compared to $89.1 \%$ in TAPS ${ }^{15}$. The other two minor cytosine modifications $5 \mathrm{fC}$ and $5 \mathrm{caC}$ also showed high conversion rate $(84.9 \%$ and $94.4 \%$ respectively, Supplementary Table 1); however, they were ignored in following data analysis due to the negligible amounts existed in the mammalian genome $(<0.002 \% \text { of total cytosine })^{4}$. Similar to TAPS ${ }^{15}$, TAPS $\beta$ showed excellent sequencing quality scores at cytosine/guanine (Supplementary Fig. 1). We observed good correlation between TAPS $\beta$ and published $5 \mathrm{mC}$ data of mESCs by reduced representation oxBS-seq (RRoxBS-seq) ${ }^{21}$ (Pearson's $r=0.77$, Fig. 1d) and wholegenome oxBS-seq 22 (Pearson's $r=0.72$, Fig. 1e). In comparison, Pearson correlation coefficients between the reported four RRoxBS-seq replicates were $0.79-0.80$ (ref. ${ }^{21}$ ). The discrepancy between TAPS $\beta$ and oxBS-seq is likely caused by biological differences, e.g. cell line passages ${ }^{9}$, rather than systematic bias, given the high correlation between TAPS and TAPS $\beta$ using the same biological sample (Supplementary Fig. 2). TAPS $\beta$ showed much improved sequencing quality evidenced by higher mapping rate (90.7\%, Supplementary Table 2) than RRoxBS-seq ${ }^{21}(66.2-68.2 \%)$ and oxBS-seq $22(21.4-26.1 \%)$. Notably, TAPS $\beta$ is the first and only bisulfite-free, base-resolution, and quantitative $5 \mathrm{mC}$-specific sequencing method.

CAPS for bisulfite-free $5 \mathrm{hmC}$-specific sequencing. To enable $5 \mathrm{hmC}$-specific sequencing, we turned to chemical oxidization of $5 \mathrm{hmC}$ to $5 \mathrm{fC}$, which can also be converted to DHU by borane reduction (Fig. 2a). In our proof-of-principle study, we used potassium perruthenate $\left(\mathrm{KRuO}_{4}\right)$ previously used in oxBS-seq as the oxidant, which is known to cause DNA damage9. In this study, we utilized potassium ruthenate $\left(\mathrm{K}_{2} \mathrm{RuO}_{4}\right)$, which was used in chemical-assisted C-to- $\mathrm{T}$ conversion of $5 \mathrm{hmC}$ sequencing (hmC-CATCH) and reported to be more oxidative and less DNA damaging than $\mathrm{KRuO}_{4}$ (ref. ${ }^{23}$ ). We optimized the $\mathrm{K}_{2} \mathrm{RuO}_{4}$ oxidation protocol for CAPS as follows: (1) Commonly used doublestrand DNA library preparation method was applied instead of the complicated single-strand protocol. (2) A uracil-containing loop-structured NEBNext Adaptor was used in the DNA ligation. Subsequent treatment with USER enzyme (a mix of UDG and Endo VIII) opened the loop, leaving $3^{\prime}$ and $5^{\prime}$ phosphate ends that could protect the ligated DNA from oxidative damaging 24 . (3) Double oxidation was performed on the ligated DNA by adding additional oxidant to the original oxidation reaction, 
a<smiles>Cc1cnc(=O)[nH]c1N</smiles>

DNA

$5 \mathrm{mC}$<smiles>Nc1nc(=O)n([N+](=O)[O-])cc1CO</smiles>

$5 \mathrm{hmC}$<smiles></smiles>

DNA

$5 \mathrm{mC}$<smiles>C1C[GeH2]C[GeH2]1</smiles>

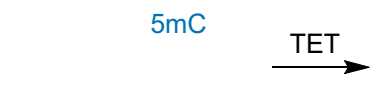<smiles>Nc1nc(=O)n([N+](=O)[O-])cc1C(=O)O</smiles><smiles>Nc1nc(=O)n(S(N)(=O)=O)cc1CCCl</smiles>

$5 \mathrm{gmC}$<smiles>C1CCCCC1</smiles>

b

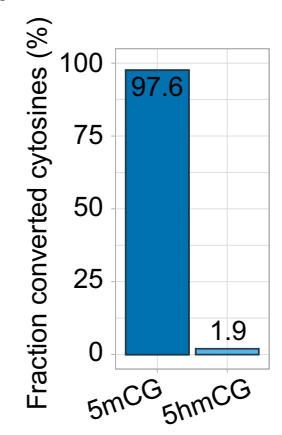

C

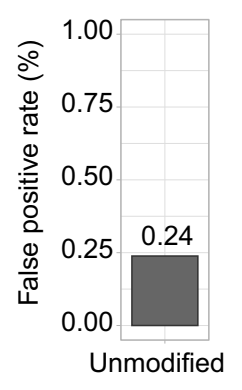

d

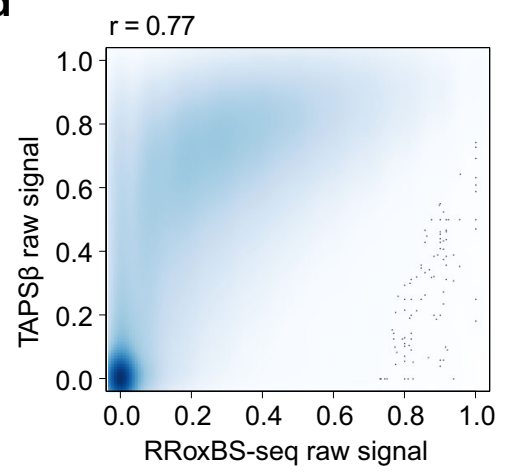<smiles>O=C1CCNC(=O)N1</smiles>

DNA

DHU

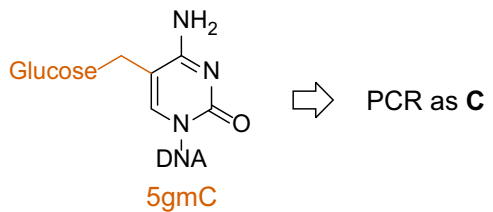

Fig. 1 TAPS $\beta$ for bisulfite-free $\mathbf{5 m C}$-specific sequencing. a Schematic demonstration of TAPS $\beta$. b Conversion rates of TAPS $\beta$ at known $5 \mathrm{mCG}$ or $5 \mathrm{hmCG}$ positions from CpG-methylated lambda DNA or synthetic spike-in. c False-positive rate of TAPS $\beta$ from 2-kb-unmodified spike-in. $\mathbf{d}$ Correlation analysis between TAPS $\beta$ and published RRoxBS-seq dataset at CpGs with the minimal depth of 10 . The color scale represents density. e Correlation analysis between TAPS $\beta$ and published oxBS-seq dataset at CpGs with the minimal depth of 10. The Pearson's $r$ is shown at the top. The raw signal for each CpG was calculated as the ratio between $C$ and the sum of $C$ and $T$.

a

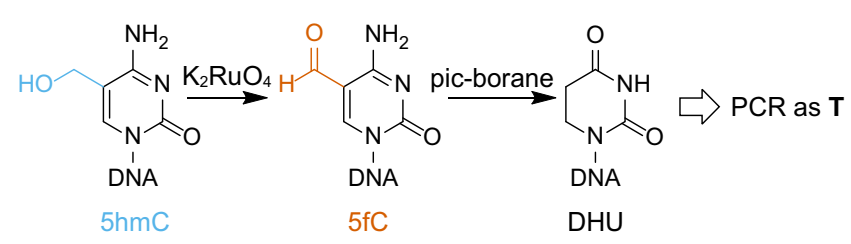

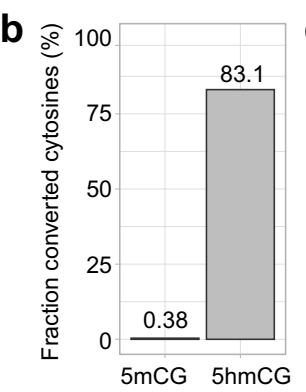

e

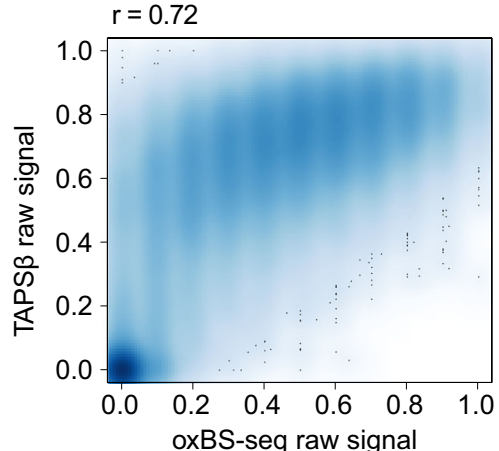


a
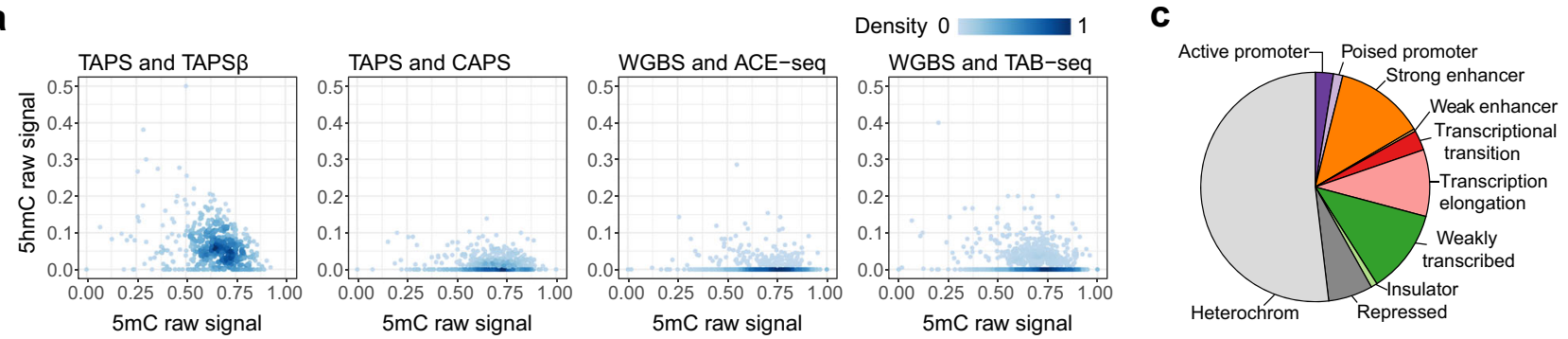

b

chr4: $126,725,380-126,726,250$

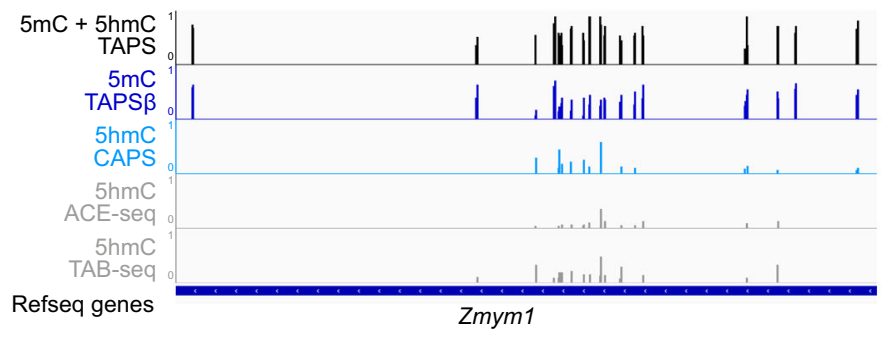

d

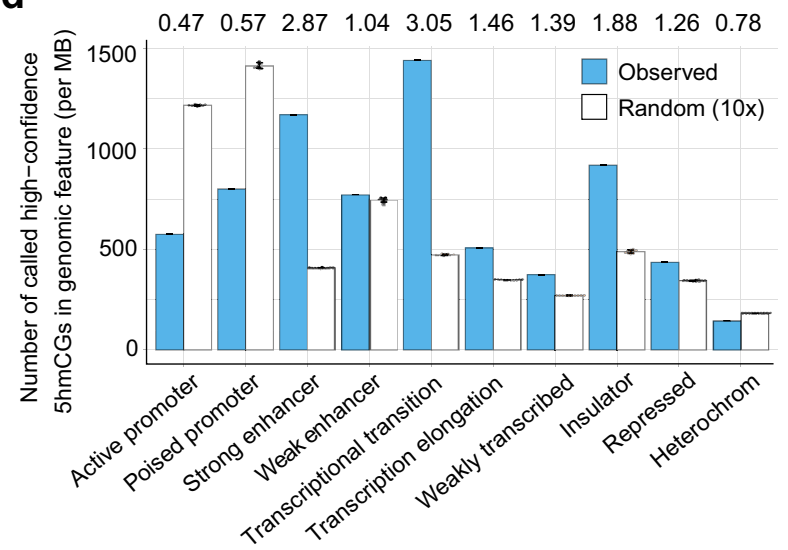

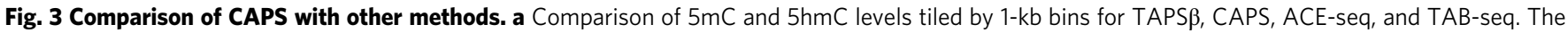

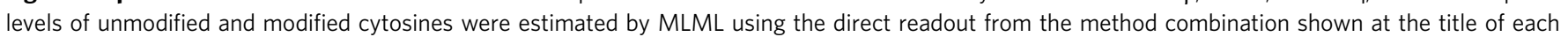

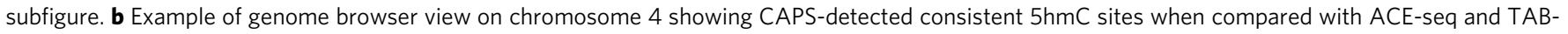

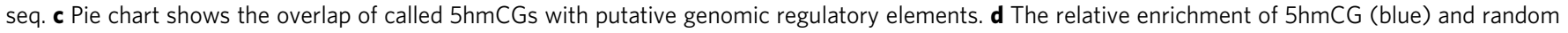

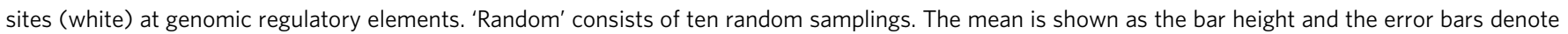

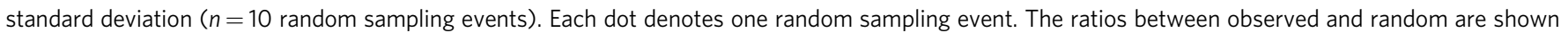
at the top.

improving the conversion rate of $5 \mathrm{hmC}$ to $5 \mathrm{fC}$ from $82.8 \%$ to 97.2\% measured by HPLC-MS/MS (Supplementary Fig. 3). A limitation of both $\mathrm{KRuO}_{4}$ and $\mathrm{K}_{2} \mathrm{RuO}_{4}$ oxidation is that they only work on single-strand DNA. Pyridine borane used in TAPS, which is optimized based on double-strand DNA, only showed $65.8 \% 5 \mathrm{hmC}$-to- $\mathrm{T}$ conversion rate on single-strand DNA while $1.3 \%$ C-to-T false-positive rate was observed (Supplementary Fig. 4). Instead, we found that another compound, 2methylpyridine borane (pic-borane), achieved $83.1 \%$ 5hmC-to$\mathrm{T}$ conversion rate (Fig. $2 \mathrm{~b}$ ) and $0.72 \%$ false-positive rate on single-strand DNA (Fig. 2c), and therefore was chosen as the reducing agent for CAPS. These numbers are similar to the $5 \mathrm{hmC}$-to- $\mathrm{T}$ conversion rate and false-positive rate reported in hmC-CATCH ( $80 \%$ (without pull-down) and $0.6-1 \%$, respectively $)^{23}$.

Next, we applied CAPS on mESCs and detected 1,762,287 5hmC-modified sites. We compared CAPS with the other two whole-genome base-resolution $5 \mathrm{hmC}$ sequencing methods: $\mathrm{TAB}-$ seq $^{10}$ and ACE-seq ${ }^{13}$, using published sequencing data from mESCs. Both TAB-seq and ACE-seq utilize $\beta$ GT to protect $5 \mathrm{hmC}$ with a glucose from bisulfite or enzymatic deamination and read it as $\mathrm{C}$ after PCR amplification, while converting both unmodified $\mathrm{C}$ and $5 \mathrm{mC}$ to $\mathrm{T}$. Bisulfite-based TAB-seq shares the same drawbacks as BS, while ACE-seq partially solves the problem by replacing the harsh chemical reaction with mild APOBEC3A enzymatic deamination. However, ACE-seq still suffers from reduced sequence complexity in the converted genome, which results in low mapping rate (Fig. $2 \mathrm{~d}$ and Supplementary Table 2), low base quality (Supplementary Fig. 5), and uneven coverage
(Supplementary Fig. 6). The low base quality in ACE-seq is caused by the unbalanced CG content in the sequencing libraries similar to $\mathrm{WGBS}^{15}$, while CAPS avoids depletion of cytosines leading to optimal data quality similar to TAPS and TAPS $\beta$. CAPS outperformed TAB-seq and ACE-seq in these sequencing metrics (Fig. 2d and Supplementary Table 2), while showing good correlation with published dataset (Pearson's $r=0.79$ with TABseq and 0.67 with ACE-seq, Fig. 2e). On the other hand, $5 \mathrm{hmC}$ obtained from TAPS-TAPS $\beta$ subtraction showed an abnormal distribution of modification levels with lower correlation (Pearson's $r=0.54$ with TAB-seq and 0.40 with ACE-seq, Fig. 2f), demonstrating that the subtraction-free method is superior for $5 \mathrm{hmC}$ profiling, especially given that $5 \mathrm{hmC}$ exists in much lower abundance than $5 \mathrm{mC}$ in most non-neuronal tissues and cell lines ${ }^{4}$, including mESCs (Supplementary Fig. 3a).

To globally benchmark different methods by accounting for $5 \mathrm{mC}$ and $5 \mathrm{hmC}$ states in mESCs, we established the abundance of both modifications (Fig. 3a). Combination of TAPS $\beta$ and CAPS showed a similar pattern to whole-genome BS (WGBS) with TAB-seq or ACE-seq while TAPS-TAPS $\beta$ subtraction overestimated $5 \mathrm{hmC}$ sites. Examples were plotted to show results from different approaches, demonstrating that CAPS detected consistent 5hmC sites with TAB-seq and ACE-seq (Fig. $3 \mathrm{~b}$ and Supplementary Fig. 7). The distribution of $5 \mathrm{hmC}$ varied across genomic regulatory elements (Fig. 3c) ${ }^{25-27}$, with particular enrichment at enhancers and insulators ${ }^{28}$, where CTCF-binding sites were enriched (Fig. 3d). This result is consistent with previous findings that $5 \mathrm{hmCs}$ are enriched in enhancers and CTCF-binding sites ${ }^{10,23}$. 
a

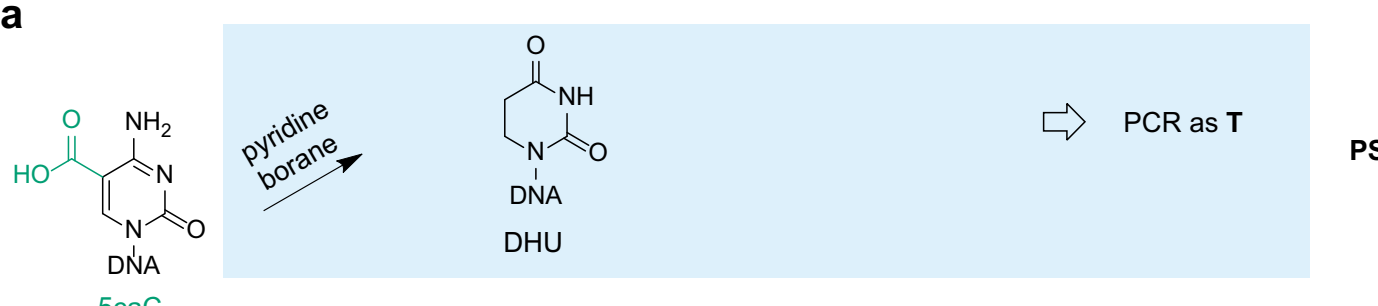

PS<smiles>Nc1nc(=O)n(O)cc1C=O</smiles>

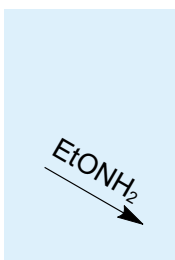<smiles></smiles><smiles>CCON=Cc1cn([N+](=O)[O-])c(=O)nc1N</smiles>

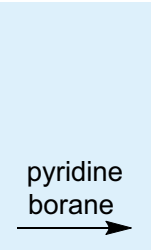<smiles>O=C1CCNC(=O)C1</smiles><smiles></smiles>

PCR as T

DNA

DHU

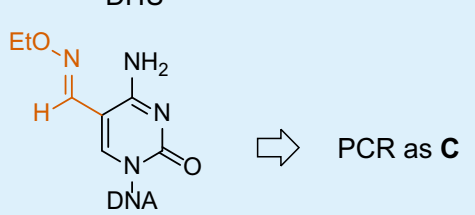

5oximeC

5oximeC

PS-c

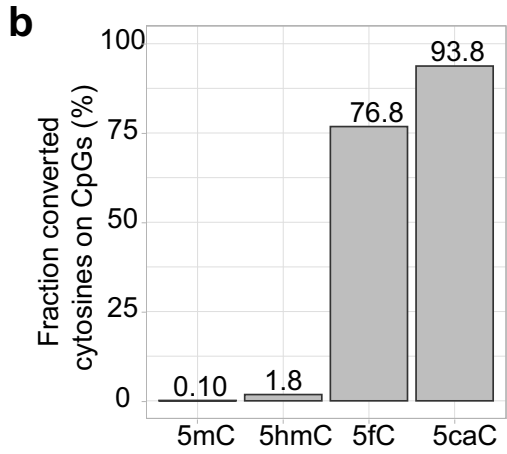

C

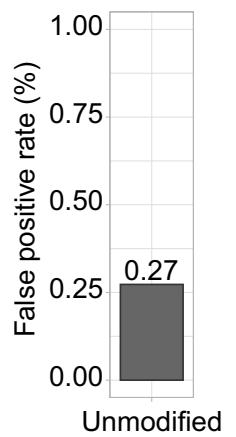

f

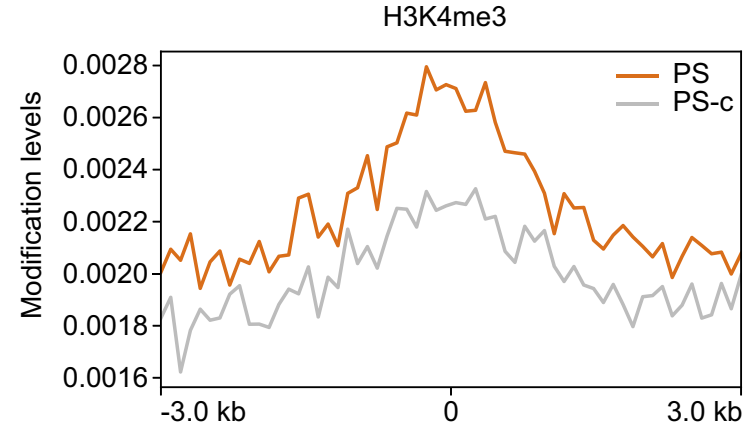

d

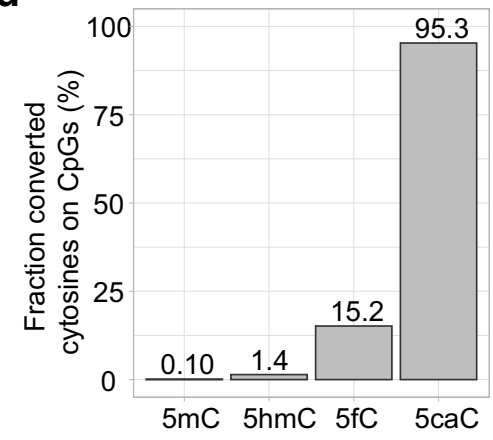

e

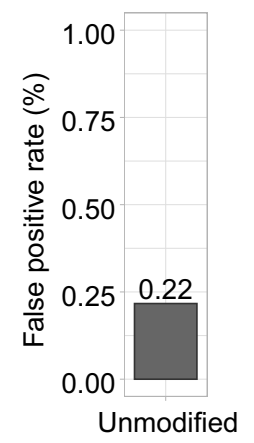

g

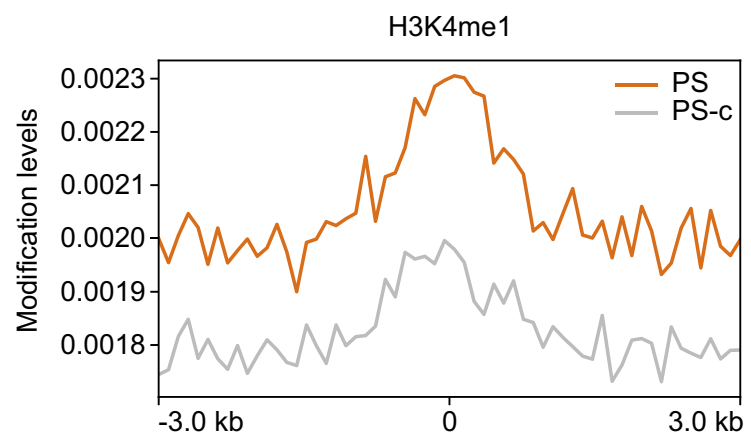

Fig. 4 PS for bisulfite-free $\mathbf{5 f C} / \mathbf{5} \mathbf{c a C}$-specific sequencing. a Schematic demonstration of PS and PS-c. b Conversion rate of PS at known $5 \mathrm{mC}, 5 \mathrm{hmC}, 5 f C$, and $5 \mathrm{caC}$ positions in spike-in controls. $\mathbf{c}$ False-positive rate of PS from 2-kb-unmodified spike-in. $\mathbf{d}$ Conversion rate of PS-c at known $5 \mathrm{mC}, 5 \mathrm{hmC}, 5 \mathrm{fC}$, and $5 \mathrm{caC}$ positions in spike-in controls. e False-positive rate of PS-c from 2-kb-unmodified spike-in. $\mathbf{f}$, $\mathbf{g}$ Average $5 \mathrm{fC} / 5 \mathrm{caC}$ signal in the 6-kb regions flanking the centers of H3K4me3 (f) and H3K4me1 (g) peaks produced by the ENCODE Project Consortium. On the x-axis, 0 denotes the peak center.

PS for bisulfite-free $\mathbf{5 f C} / 5 \mathbf{c a C}$-specific sequencing. To study the active demethylation pathway, various BS-based ${ }^{21,29-31}$ and bisulfite-free ${ }^{32}$ methods have also been developed to profile $5 \mathrm{fC}$ and/or $5 \mathrm{caC}$ modifications. The borane reduction chemistry can be used for direct sequencing of $5 \mathrm{fC}$ and $5 \mathrm{caC}$, where $5 \mathrm{fC}$ and $5 \mathrm{caC}$ are converted to $\mathrm{DHU}$ by pyridine borane and read as $\mathrm{T}$ after PCR amplification (PS, Fig. 4a). We applied this simple approach to the same mESCs gDNA and demonstrated high conversion rate in $5 \mathrm{caC}$ spike-in (93.8\%, Fig. 4b) and good conversion rate in $5 \mathrm{fC}$ spike-in (76.8\%). The low false-positive rate $(0.27 \%$, Fig. $4 \mathrm{c})$ in PS lowered the requirement for sequencing depth to distinguish the low abundant $5 \mathrm{fC} / 5 \mathrm{caC}$ signals from the background ${ }^{33}$. We also developed a method for $5 \mathrm{caC}$-specific sequencing in which $5 \mathrm{fC}$ was blocked by $\mathrm{O}$-ethylhydroxylamine ${ }^{29}$ before borane reduction reaction, which we termed pyridine borane sequencing for $5 \mathrm{caC}$ (PS-c, Fig. 4a). PS-c achieved a low conversion rate of $15.2 \%$ on $5 \mathrm{fC}$ (Fig. $4 \mathrm{~d}$ ) while the high conversion rate on $5 \mathrm{caC}(95.3 \%)$ and the low false-positive rate 


\begin{tabular}{|c|c|c|c|c|c|}
\hline Base & TAPS & TAPS $\beta$ & CAPS & PS & PS-c \\
\hline C & $C$ & C & C & C & C \\
\hline $5 \mathrm{mC}$ & $\mathbf{T}$ & $\mathbf{T}$ & C & C & C \\
\hline $5 \mathrm{hmC}$ & $\mathbf{T}$ & C & $\mathbf{T}$ & $C$ & $C$ \\
\hline $5 f C$ & $\mathrm{~T}$ & T & T & $\mathbf{T}$ & C \\
\hline $5 \mathrm{caC}$ & $\mathrm{T}$ & T & T & $\mathbf{T}$ & $\mathbf{T}$ \\
\hline
\end{tabular}

$(0.22 \%$, Fig. $4 \mathrm{e})$ were not affected. Even with the low false-positive rates of PS and PS-c, it remains challenging to detect $5 \mathrm{fC}$ and $5 \mathrm{caC}$ in whole-genome sequencing due to their low level. Instead, we focused on regulatory regions $\mathrm{s}^{27,34}$ and found that $5 \mathrm{fC} / 5 \mathrm{caC}$ signals were enriched at H3K4me1, H3K4me3 regions (Fig. 4f, g), promoters and enhancers compared to repressed regions or heterochromatin (Supplementary Fig. 8), which is consistent with previous enrichment-based $5 \mathrm{fC}$ sequencing result ${ }^{29}$. Our baseresolution data also revealed the $5 \mathrm{fC} / 5 \mathrm{caC}$ modification on Nanog, a pluripotency regulator (Supplementary Fig. 9), which was previously reported based on an enrichment-based method ${ }^{32}$. These results suggest that PS can capture the genuine $5 \mathrm{fC} / 5 \mathrm{caC}$ signals even in a low $5 \mathrm{fC}$ level sample.

Bisulfite and various bisulfite-free methods are known to have systematic differences in sequencing due to different chemistries and detection mode (indirect vs direct) ${ }^{14,15}$. When comparing various modifications, it is therefore ideal to have them derived from the same family of methods to minimize bias. In this study, we present a suite of borane reduction chemistry-based methods for direct and quantitative sequencing of all four cytosine modifications in mESCs, providing a valuable resource for studying DNA modifications in the popular epigenetics model (Table 1). By replacing harsh bisulfite treatment with mild borane reduction reaction, we achieved higher sequencing quality and more comprehensive methylome analysis. Independent identification of $5 \mathrm{mC}$ and $5 \mathrm{hmC}$ by subtraction-free TAPS $\beta$ and CAPS methods could provide insight into the distribution and function of the two modifications. The simple and mild borane reduction sequencing methods PS and PS-c could facilitate studies of the dynamics of active DNA demethylation processes. Together, they offer the most comprehensive solution for epigenetic sequencing of cytosine modifications.

\begin{abstract}
Methods
Preparation of spike-in DNA. CpG-methylated lambda DNA was produced from unmethylated lambda DNA (Promega) with M.SssI enzyme (NEB) treatment. 2-kb-unmodified spike-in was produced by PCR amplification from the pNIC28Bsa4 plasmid (Addgene, cat no. 26103). Synthetic spike-in with $5 \mathrm{mC}$ and $5 \mathrm{hmC}$ modifications was produced by annealing and extension of one oligo containing $5 \mathrm{mC}$ and another oligo containing $5 \mathrm{hmC}$ (IDT). $5 \mathrm{fC}$ spike-in was produced by an annealing and extension method with 5-formylcytidine-5'-triphosphate (5-fCTP, TriLink BioTechnologies). $5 \mathrm{caC}$ spike-in was produced by PCR amplification from the pNIC28-Bsa4 plasmid, then methylated with M.SssI enzyme (NEB) and oxidized with two rounds of mTet1CD treatment. Detailed preparation protocols and sequences of the spike-in DNA can be found in previous publication ${ }^{15}$ and Supplementary Table 3 .
\end{abstract}

mESCs culture and gDNA extraction. E14 mESCs were gifted from Professor Skirmantas Kriaucionis and cultured on gelatin-coated plates in Dulbecco's Modified Eagle Medium (DMEM) (Invitrogen) supplemented with 15\% FBS (GIBCO), $2 \mathrm{mM}$ L-glutamine (Gibco), 1\% non-essential amino acids (Gibco), 1\% penicillin/streptavidin (Gibco), $0.1 \mathrm{mM} \beta$-mercaptoethanol (Sigma), 1000 units $/ \mathrm{mL}$ LIF (Millipore), $1 \mu \mathrm{M}$ PD0325901 (Stemgent), and $3 \mu \mathrm{M}$ CHIR99021 (Stemgent). mESCs were maintained at $37^{\circ} \mathrm{C}$ and $5 \% \mathrm{CO}_{2}$ and passaged every 2 days. The gDNA was prepared by cell harvesting with centrifugation for 5 min at $1000 \times g$ and room temperature, and DNA extraction with Quick-DNA Plus kit (Zymo Research) according to the manufacturer's protocol.
Expression and purification of $\mathbf{m T e t 1 C D}$. mTet1CD was expressed in Expi293F cells from mTet1CD insert (NM_001253857.2, 4371-6392) with N-terminal Flagtag cloned into pcDNA3-Flag between KpnI and BamH1 restriction sites ${ }^{15}$. The cells were grown for $48 \mathrm{~h}$ at $37^{\circ} \mathrm{C}, 170$ r.p.m., and $5 \% \mathrm{CO}_{2}$, then harvested by centrifugation, re-suspended in the lysis buffer containing $50 \mathrm{mM}$ Tris-Cl pH 7.5, $500 \mathrm{mM} \mathrm{NaCl}, 1 \times$ cOmplete Protease Inhibitor Cocktail, $1 \mathrm{mM}$ PMSF, $1 \%$ Triton $\mathrm{X}-100$, and incubated on ice for $20 \mathrm{~min}$. The cell lysate was then clarified by centrifugation for $30 \mathrm{~min}$ at $30,000 \times g$ and $4{ }^{\circ} \mathrm{C}$. ANTI-FLAG M2 Affinity Gel was used to purify the supernatant and eluted with buffer containing $20 \mathrm{mM}$ HEPES $\mathrm{pH}$ 8.0, $150 \mathrm{mM} \mathrm{NaCl}, 0.1 \mathrm{mg} / \mathrm{mL} 3 \times$ Flag peptide, $1 \times$ cOmplete Protease Inhibitor Cocktail, $1 \mathrm{mM}$ PMSF. The collected fractions were concentrated and buffer exchanged to the final buffer containing $20 \mathrm{mM}$ HEPES pH $8.0,150 \mathrm{mM} \mathrm{NaCl}$, and $1 \mathrm{mM}$ dithiothreitol, then mixed with glycerol $(30 \% \mathrm{v} / \mathrm{v})$ for storage at $-80^{\circ} \mathrm{C}$.

Preparation of mESCs gDNA and sequencing library construction. mESCs gDNA was spiked with $0.5 \%$ of methylated lambda DNA, $0.025 \%$ of $2-\mathrm{kb}$ unmodified and $0.025 \%$ of 2 -kb-caC spike-in controls. For CAPS approach, gDNA was fragmented by Covaris M220 instrument and size-selected to 200-400 bp using Ampure XP beads (Beckman Coulter). For other approaches, gDNA was fragmented and size-selected to $300-500 \mathrm{bp} ; 0.01 \%$ of synthetic oligo with $\mathrm{N} 5 \mathrm{mCNN}$ $\mathrm{N} 5 \mathrm{hmCNN}$ sequences and $0.01 \%$ of synthetic oligo with $5 \mathrm{fC}$ modifications were added after size-selection. One-hundred nanograms of fragmented DNA was used for end-repair/A-tailing and ligation of NEBNext Adaptor (NEB) with KAPA Hyper kit (KAPA) according to the manufacturer's protocol. The uracil in the loop of NEBNext Adaptor was removed by adding $3 \mu \mathrm{L}$ of USER enzyme (NEB) to the ligation reaction and incubating for $15 \mathrm{~min}$ at $37^{\circ} \mathrm{C}$. Then the reaction was purified with $0.8 \times$ Ampure XP beads according to the manufacturer's protocol. For CAPS approach, $80 \%$ acetonitrile: $\mathrm{H}_{2} \mathrm{O}$ was used instead of $80 \%$ ethanol: $\mathrm{H}_{2} \mathrm{O}$ during the beads purification step

TAPS with $\boldsymbol{\beta}$ GT blocking (TAPS $\beta$ ). Ligated DNA was added to a $50-\mu \mathrm{L}$ reaction containing $50 \mathrm{mM}$ HEPES buffer (pH 8), $25 \mathrm{mM} \mathrm{MgCl}_{2}, 200 \mu \mathrm{M}$ UDP-Glc (NEB), and $10 \mathrm{U}$ of $\beta \mathrm{GT}$ (Thermo Fisher) for $1 \mathrm{~h}$ at $37^{\circ} \mathrm{C}$. 5hmC-blocked DNA was purified with Ampure XP and then incubated in $50 \mu \mathrm{L}$ oxidation reaction containing $50 \mathrm{mM}$ HEPES buffer (pH 8.0), $100 \mu \mathrm{M}$ ammonium iron (II) sulfate, $1 \mathrm{mM}$ a-ketoglutarate, $2 \mathrm{mM}$ ascorbic acid, $1 \mathrm{mM}$ dithiothreitol, $100 \mathrm{mM} \mathrm{NaCl}, 1.2 \mathrm{mM}$ ATP, and $4 \mu \mathrm{M}$ mTet $1 \mathrm{CD}$ for $80 \mathrm{~min}$ at $37^{\circ} \mathrm{C}$. Then $0.8 \mathrm{U}$ of Proteinase K (NEB) was added to the reaction and incubated for $1 \mathrm{~h}$ at $50^{\circ} \mathrm{C}$. Oxidized DNA was purified with Ampure XP beads and then input into another round of TET oxidation in order to achieve complete oxidation. The double-oxidized DNA was added to a $50-\mu \mathrm{L}$ reaction containing $600 \mathrm{mM} \mathrm{NaAc}(\mathrm{pH}=4.3)$ and $1 \mathrm{M}$ pyridine borane (Alfa Aesar). The reaction was incubated at $37^{\circ} \mathrm{C}$ and 850 r.p.m. in a ThermoMixer (Eppendorf) for $16 \mathrm{~h}$ and purified by Zymo-IC column (Zymo Research) with Oligo Binding Buffer (Zymo Research).

Chemical-assisted pyridine borane sequencing (CAPS). Potassium ruthenate $\left(\mathrm{K}_{2} \mathrm{RuO}_{4}\right)$ was prepared as previously described by Zeng et al. ${ }^{23}$ and stored at $-20^{\circ} \mathrm{C}$ in a refrigerator as $10 \times$ oxidant; $2 \mathrm{M}$ pic-borane (Sigma) was prepared by dissolving the solid in EtOH. Before 5hmC oxidation, ligated DNA was purified with Micro Bio-Spin P-6 SSC column (Bio-Rad, washed five times with water before use). The purified DNA was denatured in $20 \mu \mathrm{L}$ solution containing $0.05 \mathrm{M}$ $\mathrm{NaOH}$ for 30 min at $37^{\circ} \mathrm{C} ; 10 \times$ oxidant was diluted to $1 \times$ with distilled water and $2.5 \mu \mathrm{L}$ of $1 \times$ oxidant was added to the denatured DNA. The oxidation reaction was incubated at $37^{\circ} \mathrm{C}$ and 850 r.p.m. in a ThermoMixer for $1 \mathrm{~h}$. Then additional $2.5 \mu \mathrm{L}$ of $1 \times$ oxidant was added to the same reaction and incubated at $37^{\circ} \mathrm{C}$ and 850 r.p.m. in a ThermoMixer for another hour. The oxidized DNA was purified by a Bio-Rad Micro Bio-Spin P-6 SSC column, and added to a reaction containing 0.6 M MES (Sigma, pH 5.2) and $0.2 \mathrm{M}$ pic-borane. The reaction was incubated at $37^{\circ} \mathrm{C}$ and 850 r.p.m. in a ThermoMixer for $2 \mathrm{~h}$ and purified by Zymo-IC column with Oligo Binding Buffer.

Quantification of $\mathbf{5 m C}$, $5 \mathrm{hmC}$, and $\mathbf{5 f C}$ level by HPLC-MS/MS. Control and oxidized gDNA samples were digested into nucleosides by $2 \mathrm{U}$ of Nuclease P1 (Sigma-Aldrich) and $10 \mathrm{nM}$ deaminase inhibitor erythro-9-amino- $\beta$-hexyl- $\alpha$ methyl-9H-purine-9-ethanol hydrochloride (Sigma-Aldrich) at $37^{\circ} \mathrm{C}$ for overnight and then $6 \mathrm{U}$ of alkaline phosphatase (Sigma-Aldrich) and $0.5 \mathrm{U}$ of phosphodiesterase I (Sigma-Aldrich) at $37^{\circ} \mathrm{C}$ for $3 \mathrm{~h}$. After filtering with Amicon Ultra- $0.5 \mathrm{~mL}$ $10 \mathrm{~K}$ centrifugal filters (Merck Millipore), the digested samples were subjected to a ZORBAX Eclipse Plus C18 column $\left(2.1 \times 150 \mathrm{~mm}^{2}, 1.8-\mu \mathrm{m}\right.$, Agilent). HPLC-MS/ MS analysis was carried out with 1290 Infinity LC Systems (Agilent) coupled with a 6495B Triple Quadrupole Mass Spectrometer (Agilent). Detailed HPLC-MS/MS program could be found in previous publication ${ }^{15}$.

Pyridine borane sequencing (PS). Ligated DNA was added to a $50-\mu \mathrm{L}$ reaction containing $0.6 \mathrm{M} \mathrm{NaAc}(\mathrm{pH}=4.3)$ and $1 \mathrm{M}$ pyridine borane. The reaction was incubated at $37^{\circ} \mathrm{C}$ and 850 r.p.m. in a ThermoMixer for $16 \mathrm{~h}$ and purified by Zymo-IC column with Oligo Binding Buffer. 
Pyridine borane sequencing for carboxylcytosine (PS-c). Ligated DNA was added to a $50-\mu \mathrm{L}$ reaction containing $10 \mathrm{mM} O$-ethylhydroxylamine (Aldrich) and $100 \mathrm{mM}$ MES buffer (pH 5.0). The reaction was incubated at $37^{\circ} \mathrm{C}$ and 850 r.p.m. for $4 \mathrm{~h}$ in a ThermoMixer and purified with Ampure XP beads. 5fC-blocked DNA was then added to a $50-\mu \mathrm{L}$ reaction containing $0.6 \mathrm{M} \mathrm{NaAc}(\mathrm{pH}=4.3)$ and $1 \mathrm{M}$ pyridine borane. The reaction was incubated at $37^{\circ} \mathrm{C}$ and 850 r.p.m. in a ThermoMixer for $16 \mathrm{~h}$ and purified by Zymo-IC column with Oligo Binding Buffer.

PCR amplification of converted DNA and sequencing. Converted DNA was amplified with KAPA HiFi HotStart Uracil+ ReadyMix PCR Kit (KAPA) for 4 cycles according to the manufacturer's protocol with minor modification. Dual index primers in NEBNext Multiplex Oligos for Illumina were used instead of the Library Amplification Primer Mix. The PCR product was purified with $1 \times$ Ampure XP beads and quantified with Qubit dsDNA HS Assay Kit (ThermoFisher). When starting with $100 \mathrm{ng}$ of fragmented DNA for library construction, typical final library yield should be $>30 \mathrm{nM}$ after 4 cycles of PCR amplification. Libraries were sequenced on NovaSeq 6000 (150 bp paired end) with no PhiX added.

Data preprocessing. Sequencing reads were trimmed with Trim Galore! v0.3.1 (https://www.bioinformatics.babraham.ac.uk/projects/trim_galore/) to remove adaptors and low-quality bases. Trimmed reads were mapped to a genome combining spike-in sequences and the mm9 mouse genome using BWA mem v.0.7.12 (ref. ${ }^{35}$ ). PCR duplicates were removed using MarkDuplicate function of Picard v2.3.0 (http://broadinstitute.github.io/picard/). Reads with MAPQ $<10$ were excluded from methylated site calling. Modified bases were called by asTair v3.3.1 (ref. ${ }^{15}$ ). Raw signals were calculated as the ratio between $\mathrm{C}$ and $\mathrm{C}+\mathrm{T}$ at each site. Regions known to be prone to mapping artifacts (https://sites.google.com/site/ anshulkundaje/projects/blacklists) ${ }^{36,37}$ and known single nucleotide variants (http://epigenetics.hugef-research.org/data.php) ${ }^{38}$ of the E14 cell line were used to exclude those overlapping sites from subsequent analysis. The mapping rate was calculated as the ratio between the number of properly mapped read pairs (MAPQ $>10$ ) and the number of trimmed read pairs by Samtools ${ }^{39}$. The base quality was visualized by the phred function of asTair ${ }^{15}$

Published datasets. We used the following published datasets: TAPS data and WGBS data (GSE112520) ${ }^{15}$, RRoxBS-seq data (GSM1364069) ${ }^{21}$, oxBS-seq data $\left(\right.$ GSE112875) ${ }^{22}$, TAB-seq data $(\text { GSE36173) })^{10}$, and ACE-seq data (GSE116016) ${ }^{13}$. The TAB-seq data were reprocessed to obtain the full list of modified and unmodified sites. The sequencing reads were downloaded and trimmed by Trim Galore! v0.3.1 (https://www.bioinformatics.babraham.ac.uk/projects/trim_galore/). The trimmed reads were aligned to $\mathrm{mm} 9$ using Bismark v0.18.1 (ref. ${ }^{40}$ ) and Bowtie v2.2.1 (ref. ${ }^{41}$ ). PCR duplicates were removed from the mapped bam file using MarkDuplicate function of Picard v2.3.0 (http://broadinstitute.github.io/picard/). The reads with over three non-conversion sites were filtered using the filter_non_conversion function of bismark as previously described ${ }^{10}$. The methylation sites were called by bismark methylation extractor and masked by intersectBed (Bedtools v2.25.0 $)^{42}$ to remove sites in regions known to be prone to mapping artifacts.

Pairwise comparisons of TAPS $\beta$. Replicate one of RRoxBS-seq data was used due to the highest number of reads among the four replicates ${ }^{21}$. The three replicates of whole-genome oxBS-seq 22 results were pooled together for the correlation analysis. Sites with a minimal coverage of ten reads were used for the correlation analysis between TAPS $\beta$ and oxBS-seq. The Pearson correlation coefficient (Pearson's $r$ ) was calculated by using R function cor. The scatterplots with smoothed densities color representation were visualized using function smoothScatter in R.

Coverage analysis of CAPS and ACE-seq. The CpG island annotation was downloaded from UCSC ${ }^{43}$. Each $\mathrm{CpG}$ island was evenly binned into ten windows. The 4-kb flanking regions were binned into 20 windows. The coverage was defined as the sum of modified and unmodified reads at each site. The average coverage was calculated by Bedtools map ${ }^{42}$. Given that the overall coverage of CAPS was higher than ACE-seq, the coverage at each site was normalized by the ratio of overall coverage between the two datasets.

Pairwise comparisons of CAPS. To compare CAPS with ACE-seq and TAB-seq, the raw $5 \mathrm{hmCG}$ signals, i.e. $\mathrm{C} /(\mathrm{C}+\mathrm{T})$, were calculated within $10 \mathrm{-kb}$ genomic bins (Fig. $2 \mathrm{f}$ ) as previously defined ${ }^{13}$. The $10-\mathrm{kb}$ raw signal of TAPS-TAPS $\beta$ subtraction was calculated as the average estimated $5 \mathrm{hmC}$ levels from the maximum likelihood methylation levels (MLML) output.

Estimation of $\mathbf{5 h m C}$ using maximum likelihood. To estimate $5 \mathrm{hmC}$ levels from TAPS and TAPS $\beta$, the MLML estimation method ${ }^{19}$ was applied on sites with a minimum coverage of 5 . The sites with at least one conflict were excluded from subsequent analysis. The average levels of $5 \mathrm{mC}$ and $5 \mathrm{hmC}$ estimated by MLML were tiled by 1-kb bins (Fig. 3a).
Genomic view. To view the methylation levels on genomes, the methylation calling output was transferred to the bigwig format by bedGraphToBigWig ${ }^{44}$ and visualized by the Integrative Genomics Viewer ${ }^{45}$ on the $\mathrm{mm} 9$ genome.

Statistical test of $\mathbf{5 h m C}$. We used the binomial test ${ }^{10}$ to call $5 \mathrm{hmC}$ at sites with the minimal coverage of five reads. The probability $p$ of the binomial distribution was the false-positive rate (0.0072) of CAPS, calculated from the unmodified control DNA (Fig. 2c). Cytosines with Benjamini-Hochberg (BH) adjusted $p$-value $<0.05$ were used for downstream analysis.

Quantifying enrichment of 5hmCGs in regulatory elements. The list of putative genomic regulatory elements was downloaded (https://github.com/gireeshkbogu/ chromatin_states_chromHMM_mm9 $)^{27}$. This list was predicted based on the ENCODE data ${ }^{26}$ by ChromHMM ${ }^{25}$. The high-confidence $5 \mathrm{hmCG}$ sites (BHadjusted $p$-value $<0.05$ and coverage $\geq 5$ reads) were annotated using bedtools intersect. The number of 5hmCG sites falling into each category was counted (Fig. 3c). To investigate the enrichment of 5 hmCG in each element class, a set of CG sites was sampled for ten times to generate a background distribution of CG sites across element categories. The number of $5 \mathrm{hmCGs}$ or random CGs was normalized by the genomic coverage of corresponding regulatory elements.

Genome-wide analysis of PS and PS-c. The histone modification ChIP-seq data were downloaded from the ENCODE project ${ }^{34}$ : H3K4mel (GSM1000121) and H3K4me3 (GSM1000124). The prediction result of genomic regulatory elements ${ }^{27}$ was downloaded as described above. The centers of broad peaks or predicted regions were used. Average modification levels were calculated by tiling the left and right flanking $3 \mathrm{~kb}$ regions into 100 -bp bins. The profiles were visualized by deepTools 3.3.0 (ref. ${ }^{46}$ ).

Reporting summary. Further information on research design is available in the Nature Research Reporting Summary linked to this article.

\section{Data availability}

All sequencing data of this study are deposited at the Gene Expression Omnibus (accession: GSE155613). Published data used in this study include TAPS data and WGBS data (GSE112520) $)^{15}$, RRoxBS-seq data (GSM1364069) ${ }^{21}$, oxBS-seq data $(\text { GSE112875) })^{22}$, TAB-seq data (GSE36173) ${ }^{10}$ and ACE-seq data (GSE116016) ${ }^{13}$, H3K4mel ChIP-seq data (GSM1000121), and H3K4me3 ChIP-seq data (GSM1000124) ${ }^{34}$. All relevant additional data have been published with the manuscript, either as part of the main text or in the supplement.

\section{Code availability}

The analysis scripts are available at https://github.com/zhiyhu/CAPS-paper; https://doi. org/10.5281/zenodo.4321048 (ref. ${ }^{47}$ ).

Received: 26 August 2020; Accepted: 21 December 2020; Published online: 27 January 2021

\section{References}

1. Li, E. \& Zhang, Y. DNA methylation in mammals. Cold Spring Harb. Perspect. Biol. 6, a019133 (2014).

2. Tahiliani, M. et al. Conversion of 5-methylcytosine to 5hydroxymethylcytosine in mammalian DNA by MLL partner TET1. Science 324, 930-935 (2009).

3. Kriaucionis, S. \& Heintz, N. The nuclear DNA base 5-hydroxymethylcytosine is present in Purkinje neurons and the brain. Science 324, 929-930 (2009).

4. Ito, S. et al. Tet proteins can convert 5-methylcytosine to 5-formylcytosine and 5-carboxylcytosine. Science 333, 1300-1303 (2011).

5. He, Y. F. et al. Tet-mediated formation of 5-carboxylcytosine and its excision by TDG in mammalian DNA. Science 333, 1303-1307 (2011).

6. Bachman, M. et al. 5-Formylcytosine can be a stable DNA modification in mammals. Nat. Chem. Biol. 11, 555-557 (2015).

7. Kellinger, M. W. et al. 5-formylcytosine and 5-carboxylcytosine reduce the rate and substrate specificity of RNA polymerase II transcription. Nat. Struct. Mol. Biol. 19, 831-833 (2012).

8. Raiber, E.-A., Hardisty, R., van Delft, P. \& Balasubramanian, S. Mapping and elucidating the function of modified bases in DNA. Nat. Rev. Chem. 1, 0069 (2017)

9. Booth, M. J. et al. Quantitative sequencing of 5-methylcytosine and 5hydroxymethylcytosine at single-base resolution. Science 336, 934-937 (2012).

10. $\mathrm{Yu}, \mathrm{M}$. et al. Base-resolution analysis of 5-hydroxymethylcytosine in the mammalian genome. Cell 149, 1368-1380 (2012). 
11. Tanaka, K. \& Okamoto, A. Degradation of DNA by bisulfite treatment. Bioorg. Med. Chem. Lett. 17, 1912-1915 (2007).

12. Zhao, L. Y., Song, J., Liu, Y., Song, C. X. \& Yi, C. Mapping the epigenetic modifications of DNA and RNA. Protein Cell 11, 792-808 (2020).

13. Schutsky, E. K. et al. Nondestructive, base-resolution sequencing of 5hydroxymethylcytosine using a DNA deaminase. Nat. Biotechnol. 36, 1083-1090 (2018).

14. Vaisvila, R. et al. EM-seq: detection of DNA methylation at single base resolution from picograms of DNA. Preprint at bioRxiv https://doi.org/ 10.1101/2019.12.20.884692 (2020).

15. Liu, Y. et al. Bisulfite-free direct detection of 5-methylcytosine and 5hydroxymethylcytosine at base resolution. Nat. Biotechnol. 37, 424-429 (2019).

16. Liu, Y. et al. Accurate targeted long-read DNA methylation and hydroxymethylation sequencing with TAPS. Genome Biol. 21, 54 (2020).

17. Mellen, M., Ayata, P., Dewell, S., Kriaucionis, S. \& Heintz, N. MeCP2 binds to $5 \mathrm{hmC}$ enriched within active genes and accessible chromatin in the nervous system. Cell 151, 1417-1430 (2012).

18. He, Y. et al. DeepH\&M: estimating single-CpG hydroxymethylation and methylation levels from enrichment and restriction enzyme sequencing methods. Sci. Adv. 6, eaba0521 (2020).

19. Qu, J., Zhou, M., Song, Q., Hong, E. E. \& Smith, A. D. MLML: consistent simultaneous estimates of DNA methylation and hydroxymethylation. Bioinformatics 29, 2645-2646 (2013).

20. Song, C. X. et al. Selective chemical labeling reveals the genome-wide distribution of 5-hydroxymethylcytosine. Nat. Biotechnol. 29, 68-72 (2011).

21. Booth, M. J., Marsico, G., Bachman, M., Beraldi, D. \& Balasubramanian, S. Quantitative sequencing of 5-formylcytosine in DNA at single-base resolution. Nat. Chem. 6, 435-440 (2014).

22. Liu, C. et al. DNA 5-methylcytosine-specific amplification and sequencing. J. Am. Chem. Soc. 142, 4539-4543 (2020).

23. Zeng, $H$. et al. Bisulfite-free, nanoscale analysis of 5-hydroxymethylcytosine at single base resolution. J. Am. Chem. Soc. 140, 13190-13194 (2018).

24. Wang, Y., Zhang, X., Wu, F., Chen, Z. \& Zhou, X. Bisulfite-free, single baseresolution analysis of 5-hydroxymethylcytosine in genomic DNA by chemicalmediated mismatch. Chem. Sci. 10, 447-452 (2019).

25. Ernst, J. \& Kellis, M. ChromHMM: automating chromatin-state discovery and characterization. Nat. Methods 9, 215-216 (2012).

26. Shen, Y. et al. A map of the cis-regulatory sequences in the mouse genome. Nature 488, 116-120 (2012).

27. Bogu, G. K. et al. Chromatin and RNA maps reveal regulatory long noncoding RNAs in mouse. Mol. Cell. Biol. 36, 809-819 (2015).

28. Kim, S., Yu, N. K. \& Kaang, B. K. CTCF as a multifunctional protein in genome regulation and gene expression. Exp. Mol. Med. 47, e166 (2015).

29. Song, C. X. et al. Genome-wide profiling of 5-formylcytosine reveals its roles in epigenetic priming. Cell 153, 678-691 (2013).

30. Wu, H., Wu, X., Shen, L. \& Zhang, Y. Single-base resolution analysis of active DNA demethylation using methylase-assisted bisulfite sequencing. Nat. Biotechnol. 32, 1231-1240 (2014).

31. Lu, X. et al. Base-resolution maps of 5-formylcytosine and 5-carboxylcytosine reveal genome-wide DNA demethylation dynamics. Cell Res. 25, 386-389 (2015).

32. Xia, B. et al. Bisulfite-free, base-resolution analysis of 5 -formylcytosine at the genome scale. Nat. Methods 12, 1047-1050 (2015).

33. Wu, H., Wu, X. \& Zhang, Y. Base-resolution profiling of active DNA demethylation using MAB-seq and caMAB-seq. Nat. Protoc. 11, 1081-1100 (2016).

34. Yue, F. et al. A comparative encyclopedia of DNA elements in the mouse genome. Nature 515, 355-364 (2014)

35. Li, H. \& Durbin, R. Fast and accurate short read alignment with BurrowsWheeler transform. Bioinformatics 25, 1754-1760 (2009).

36. Consortium, E. P. An integrated encyclopedia of DNA elements in the human genome. Nature 489, 57-74 (2012).

37. Amemiya, H. M., Kundaje, A. \& Boyle, A. P. The ENCODE blacklist: identification of problematic regions of the genome. Sci. Rep. 9, 9354 (2019).

38. Incarnato, D., Krepelova, A. \& Neri, F. High-throughput single nucleotide variant discovery in E14 mouse embryonic stem cells provides a new reference genome assembly. Genomics 104, 121-127 (2014).

39. Li, H. et al. The Sequence Alignment/Map format and SAMtools. Bioinformatics 25, 2078-2079 (2009).

40. Krueger, F. \& Andrews, S. R. Bismark: a flexible aligner and methylation caller for Bisulfite-Seq applications. Bioinformatics 27, 1571-1572 (2011).
41. Langmead, B. \& Salzberg, S. L. Fast gapped-read alignment with Bowtie 2. Nat. Methods 9, 357-359 (2012).

42. Quinlan, A. R. \& Hall, I. M. BEDTools: a flexible suite of utilities for comparing genomic features. Bioinformatics 26, 841-842 (2010).

43. Rosenbloom, K. R. et al. ENCODE data in the UCSC Genome Browser: year 5 update. Nucleic Acids Res. 41, D56-D63 (2013).

44. Kent, W. J., Zweig, A. S., Barber, G., Hinrichs, A. S. \& Karolchik, D. BigWig and BigBed: enabling browsing of large distributed datasets. Bioinformatics 26, 2204-2207 (2010)

45. Robinson, J. T. et al. Integrative genomics viewer. Nat. Biotechnol. 29, 24-26 (2011).

46. Ramirez, F. et al. deepTools2: a next generation web server for deepsequencing data analysis. Nucleic Acids Res. 44, W160-W165 (2016).

47. Hu, Z., Liu, Y., Cheng, J. \& Song, C. -X. Subtraction-free and bisulfite-free specific sequencing of 5-methylcytosine and its oxidized derivatives at base resolution, CAPS-paper. https://doi.org/10.5281/zenodo.4321048 (2020).

\section{Acknowledgements}

We would like to acknowledge Y. Bi for helping with the HPLC-MS/MS; M. Muers for editing the manuscript. We acknowledge the Computational Biology Research Group at the University of Oxford for their technical support. This work was funded by the Ludwig Institute for Cancer Research. C.-X. Song lab is also supported by Cancer Research UK (C63763/A26394 and C63763/A27122), National Institute for Health Research (NIHR) Oxford Biomedical Research Centre (BRC), and Emerson Collective. Z.H. and A.A.A. are supported by the NIHR Oxford BRC and Ovarian Cancer Action. The views expressed are those of the author(s) and not necessarily those of the NHS, the NIHR, or the Department of Health.

\section{Author contributions}

Y.L. and C.-X.S. conceived and designed the study. Y.L. performed the experiments with the help from P.S.-Z., J.C., and M.I. Z.H. performed the computational analysis with the help from J.C. and A.A.A. Y.L., Z.H., and C.-X.S. wrote the manuscript with the help from A.A.A.

\section{Competing interests}

C.-X.S. and Y.L. are named as inventors on pending patent applications (PCT/US2019/ 012627) filed by the Ludwig Institute for Cancer Research for the technologies described here, which have been licensed to Exact Sciences Innovation. C.-X.S. is a consultant to Exact Sciences Innovation and Y.L. is an employee at Exact Sciences Innovation. Other authors declare no competing interests.

\section{Additional information}

Supplementary information is available for this paper at https://doi.org/10.1038/s41467021-20920-2.

Correspondence and requests for materials should be addressed to C.-X.S.

Peer review information Nature Communications thanks Miguel Branco and the other, anonymous, reviewer(s) for their contribution to the peer review of this work.

Reprints and permission information is available at http://www.nature.com/reprints

Publisher's note Springer Nature remains neutral with regard to jurisdictional claims in published maps and institutional affiliations.

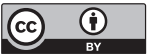

Open Access This article is licensed under a Creative Commons Attribution 4.0 International License, which permits use, sharing, adaptation, distribution and reproduction in any medium or format, as long as you give appropriate credit to the original author(s) and the source, provide a link to the Creative Commons license, and indicate if changes were made. The images or other third party material in this article are included in the article's Creative Commons license, unless indicated otherwise in a credit line to the material. If material is not included in the article's Creative Commons license and your intended use is not permitted by statutory regulation or exceeds the permitted use, you will need to obtain permission directly from the copyright holder. To view a copy of this license, visit http://creativecommons.org/ licenses/by/4.0/.

(C) The Author(s) 2021 